\title{
Kala-Azar: Nature, Biophysical Aspects, Clinical Manifestation and Treatment
}

\author{
Ersin Ismail ${ }^{1}$, Velina Bogdanova ${ }^{1}$, Georgi Shopov ${ }^{1}$, Svetoslav Dobrev $^{1}$ and Natasha Ivanova ${ }^{2}$ \\ 1. Faculty of Medicine, Medical University of Varna, Varna 9010, Bulgaria \\ 2. Department of Medical Physics and Biophysics, Faculty of Pharmacy, Medical University of Varna, Varna 9010, Bulgaria
}

\begin{abstract}
The aim of this study is to focus on a disease with a potential global health risk-VL (visceral leishmaniasis) or kala-azar. By understanding the mechanism of occurrence, development and countering infection, we can expect significant improvement in the indicators of morbidity and mortality. The specific method of transmission, as well as the necessary conditions for it, opens wide opportunities for prevention. The biophysical aspects that we present make it possible to assess the seriousness of the advancing physiological changes. This ensures adequate evaluation of the associated risks and search for an optimal therapy. We have found that from the first manifestations of kala-azar up until today, a series of countermeasures have been taken up. Acknowledging and expecting the massiveness of the infectious process, it is possible for it to cover areas whose population is unprepared to handle the contamination optimally. This requires the continuation of studies aimed at finding the most affordable and biologically tolerable medicine. The available treatment option for visceral leishmaniasis is problematic when it comes to efficacy, adverse effects and cost, making the treatment a complex issue. Not to mention toxicity of the drugs, ability to monitor side effects, length of treatment and capacity of the healthcare service to administer an accurate therapy. The main drugs available for treatment of VL (visceral leishmaniasis) are systemic agents like Antimony, Amphotericin and nowadays the oral drug Miltefosine.
\end{abstract}

Key words: Kala-azar, transmission, biophysical aspects, biologically tolerable medicine, therapy.

\section{Introduction}

Leishmaniasis is a protozoan transmitted disease. It is caused by blood flagellates that parasitize in the organs of the reticuloendothelial system and the skin, respectively visceral and cutaneous leishmaniasis. Visceral leishmaniasis is also known as kala-azar (from Hindi "black fever") due to the specific skin hyperpigmentation discovered in patients from the Hindustan Peninsula, India [1]. The problem with global warming is that it leads to increased spread of many infectious diseases, which means that some, if not most countries would be unprepared to face the consequences of an outbreak in an unusual place. Therefore, we believe that a correct understanding of the mechanism of transmission and treatment of kala-azar predisposes an expanding scientific interest

Corresponding author: Ersin Ismail, Ph.D. candidate, research fields: tropical medicine, obstetrics and gynecology, anesthesiology. in this disease and finding new universal methods of prevention and therapy.

Leishmaniasis is the second largest parasitic killer in the world (after malaria), responsible for an estimated 200,000 to 400,000 infections each year worldwide [2].

Kala-azar is an example for harmful positive feedback - an interaction between the managing body (immune system) and the object of management (bone marrow) in a biological cybernetic system. The parasite, due to its characteristics, is not degraded by enzymes in the phagocytic cell, but instead lives on, dividing mitotically.

Parasites enter the bloodstream and migrate to the internal organs, such as the liver, spleen and bone marrow and, if left untreated, almost always results in the death of the host. The most common symptoms are: fever, weight loss, fatigue, anemia, substantial swelling of the spleen and liver, skin lesions. 


\section{Contamination}

The mechanism of infection requires the obligatory participation of insects of the genus Phlebotomus. They become infected when ingesting the blood of an affected human or animal. At the end of the first day, the parasites morph into promastigotes, thus making the disease contagious for the next $7 \sim 8$ days. During this period of time, the mosquitoes can bite healthy people [3]. The incidence of kala-azar is not uniformly distributed in risk areas and it is impossible to predict the number of infected humans. The reason for this is the focal character of visceral leishmaniasis-where the outbreak occurs, how people manage disease transmission, preventing methods. Although rare, kala-azar can be congenital or befall after blood transfusion.

\section{Biophysical Aspects}

The parasite that enters the phagocytic cell is supposed to be digested by its enzymes. Because of its specific features, it is not destroyed but instead begins dividing mitotically with a rapid pace. This uncontrollable division results in the death of the host cell and breakdown of its cellular integrity. Hence, parasites are released into the bloodstream, where their presence stimulates the immune system to produce greater amounts of white blood cells from the red bone marrow. It is known that the body reacts to invaders - viruses, bacteria and the like, by stimulating hematopoiesis of leukocytes to increase their total count. This provides a high-quality response to the counter. Stabilization of the system, which is unbalanced during the disease, returns to normal the leukocyte levels.

In the pathology associated with kala-azar, harmful positive feedback is observed. The managing body (immune system) sends signals to the object of management — red bone marrow to produce a growing number of leukocytes that attack the parasites. This, however, happens at the expense of the red blood cell line which halts its proliferation. The developing erythropenia and subsequent reduction of hemoglobin in the blood cause anemia which intensifies with the advancement of the disease.

\section{Clinical Manifestation}

The first symptoms of the disease take place after a different incubation period, an average of 3 to $6 \sim 8$ months. Initial complaints are of general nature-lethargy and fatigue, loss of appetite, fever, minor increase of the spleen [4]. Further enlargement of the latter is a condition known as splenomegaly. It is explained by the fact that the spleen is an organ which a huge number of leukocytes go through before they become immunocompetent. The fever is presented in a typical form, also known as "Rogerson's type" with a characteristic twofold and threefold rise and fall during the day, especially in the Indian variation of the disease. Often peripheral lymph nodes enlarge, to a lesser degree the liver. Hematopoiesis of the erythrocyte line is suppressed, resulting in a rapidly progressing anemia.

Essential for the Indian kala-azar is the late manifestation of the infection, known as post kala-azar dermal leishmaniasis. Skin lesions contain a great amount of the parasite.

\section{Diagnosis}

Diagnosis of kala-azar includes taking material for research-peripheral blood, puncture of lymph nodes, spleen, liver or bone marrow [5]. The last has the highest diagnostic value. The most commonly used method is microscopy of the preparation, stained with Romanovsky-Giemsa. Technique: the imprint or smear is made on a slide, then it is fixed in alcohol and stained. Under the microscope the parasite has an oval shape, with a size of $2 \sim 4 \mu \mathrm{m}$, located intracellularly, sometimes up to $20 \sim 30$ in one single cell. Other methods are NNN (Novy-MacNeal-Nicolle) culture medium, serodiagnosis, etc. 


\section{Treatment}

The heavy metal antimony has been used for treatment of kala-azar since the 1940s. It comes in the form of two drugs: SSG (sodium stiboglyukonat) which contains $100 \mathrm{mg}$ antimony/100 mL (brand name Pentostam), and Meglumine antimoniate - with active ingredient $85 \mathrm{mg}$ of antimony/100 mL, also known as Glucontime. Both have similar efficiency and toxicity [6]. They have low oral bioavailability which creates the necessity of an i.v. or i.m. application. Some of the research from the 1980s shows that a dose of $20 \mathrm{mg} / \mathrm{kg} /$ day instead of $10 \mathrm{mg} / \mathrm{kg} /$ day enhances the recuperation process without there being a significant increase in toxicity. The treatment course should be continued for at least 20 days, preferably 28 . Side effects include: nausea, vomiting, joint pain, hepatitis, pancreatitis and cardiac arrhythmia.

$\mathrm{AB}$ (Amphotericin $\mathrm{B}$ ) has an excellent in vitro activity against kala-azar parasites, but its use is limited due to its toxicity. LAB (liposomal $\mathrm{AB}$ ) is of lipophilic nature, thereupon it has a higher diffusion rate which allows superior efficiency and lower toxicity with a lower dose. A research of Bichar in 2004 compares clinical efficiency of $\mathrm{AB}(1 \mathrm{mg} / \mathrm{kg} /$ day for 30 days $)$ and LAB $(2 \mathrm{mg} / \mathrm{kg} / \mathrm{day}$ for 5 days) as treatment for kala-azar patients. The results show that high levels of the drug lead to recovery in the span of 6 months ( $96 \%$ in both groups). However, side effects have also been observed, such as fever and numbness, hypokalaemia, renal dysfunction and anemia. They are more common amongst the AB-group (98\% show side effects), while $71 \%$ of the LAP-group do not show any reaction. Expectedly, average financial expenses for treatment with LAP is more than twice higher- $\$ 872$, compared to $\$ 417$ for $A B$. Mostly because of the price, using Amphotericine for kala-azar treatment is highly limited.

Miltefosine is membrane active alkyl phospholipid, primarily used as a cancer treating drug but its anti-leishmanian activity was discovered in the 1990s. Earlier investigations show that the optimal dose, which balances efficiency and tolerance, is $100 \mathrm{mg} /$ day for 28 days, taken perorally due to its good oral bioavailablity [7]. In vivo testing has demonstrated its reproductive toxicity, therefore, it can not be used by pregnant women. Other common side effects include gastrointestinal symptoms, rarely severe enough to terminate the treatment.

\section{Conclusions}

Undoubtedly, kala-azar is a disease that brings a significant global health risk, especially when facing a big outbreak or a high number of infected people. Its propagation to the untypical, more northerly situated countries would be a big challenge for their healthcare system. In our opinion, neither higher economic and social status, neither the preventative work of experts would lead to a complete and successful management of the disease. Despite the accumulated clinical experience, the concept of treating kala-azar should not be overlooked by scientists. Better understanding and responsible management of the disease will prevent at least from its underestimation.

\section{References}

[1] Bora, D. 1999. "Epidemiology of Visceral Leishmaniasis in India." Natl. Med. J. India 12: 62-8.

[2] WHO (World Health Organization). 2014. "Leishmaniasis." In WHO Fact Sheet N375. Geneva: World Health Organization.

[3] Markell, E. K. 2006. Medical Parasitology. 9th ed. Philadelphia: Saunders Ltd., Elsevier, 120-5.

[4] Farrar, J., and Hotez, P. J. 2014. Mansion's Tropical Diseases. 23rd ed. Philadelphia: Saunders Ltd., Elsevier, 638-9.

[5] Andresen, K., Gasim, S., El Hassan, A. M., Khalil, E. A., Barker, D. C., Theander, T. G., et al. 1997. "Diagnosis of Visceral Leishmaniasis by Polymerase Chain Reaction Using Blood, Bone Marrow and Lymphnode Samples from Patients from the Sudan." Trop. Med. Int. Health 2: 440-4.

[6] Chulay, J. D., Fleckenstein, L., and Smith, D. H. 1988. "Pharmokinetics of Antimony during Treatment of Visceral Leishmaniaisis with Sodium Stibogluconate or Meglumine Antimoniate." Trans. R. Soc. Trop. Med. Hyg. 82: 69-72. 
[7] Sundar, S., Mehta, H., Suresh, A. V., Singh, S. P., Rai, M., and Murray, H. W. 2004. "Amphotericin B Treatment for
Indian Visceral Leishmaniasis: Conventional Versus Lipid Formulations." Clin. Infect. Dis. 38: 377-83. 